\title{
Plastic surgeons' self-reported operative infection rates at a Canadian academic hospital
}

\author{
Wendy KY Ng MD ${ }^{1}$, Manraj Nirmal Kaur MSc ${ }^{1,2,3}$, Achilleas Thoma MD MSc FRCSC FACS 1,2,4
}

WKY Ng, MN Kaur, A Thoma. Plastic surgeons' self-reported operative infection rates at a Canadian academic hospital. Plast Surg 2014;22(4):237-240.

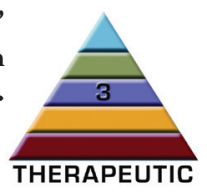

BACKGROUND: Surgical site infection rates are of great interest to patients, surgeons, hospitals and third-party payers. While previous studies have reported hospital-acquired infection rates that are nonspecific to all surgical services, there remain no overall reported infection rates focusing specifically on plastic surgery in the literature.

OBJECTIVE: To estimate the reported surgical site infection rate in plastic surgery procedures over a 10 -year period at an academic hospital in Canada.

METHODS: A review was conducted on reported plastic surgery surgical site infection rates from 2003 to 2013, based on procedures performed in the main operating room. For comparison, prospective infection surveillance data over an eight-year period (2005 to 2013) for nonplastic surgery procedures were reviewed to estimate the overall operative surgical site infection rates.

RESULTS: A total of 12,183 plastic surgery operations were performed from 2003 to 2013, with 96 surgical site infections reported, corresponding to a net operative infection rate of $0.79 \%$. There was a $0.49 \%$ surgeonreported infection rate for implant-based procedures. For non-plastic surgery procedures, surgical site infection rates ranged from $0.04 \%$ for cataract surgery to $13.36 \%$ for high-risk abdominal hysterectomies.

DISCUSSION: The plastic surgery infection rate at the study institution was found to be $<1 \%$. This rate was equal to, or somewhat less than, surgical site infection rates. However, these results do not report patterns of infection rates germane to procedures, season, age groups or sex. To provide more in-depth knowledge of this topic, multicentre studies should be conducted.

Key Words: Infections rates; Plastic surgery procedures; Surgical procedures

\section{Le taux d'infections opératoires déclaré par les plasticiens dans les hôpitaux universitaires canadiens}

HISTORIQUE : Les patients, les chirurgiens, les hôpitaux et les tiers payeurs s'intéressent au taux d'infections au site opératoire. Des études antérieures ont fait état du taux d'infections nosocomiales non spécifiques dans tous les services chirurgicaux, mais les publications ne rendent pas compte du taux global d'infections attribuables exclusivement à la chirurgie plastique.

OBJECTIF : Estimer le taux d'infections déclaré au site opératoire en chirurgie plastique sur une période de dix ans dans un hôpital universitaire du Canada.

MÉTHODOLOGIE : Les chercheurs ont effectué une analyse du taux d'infections au site opératoire en chirurgie plastique entre 2003 et 2013, d'après les interventions effectuées au bloc opératoire principal. À titre comparatif, ils ont examiné les données prospectives de surveillance des infections colligées sur une période de huit ans (2005 à 2013) après des interventions non chirurgicales pour évaluer le taux global d'infections au site opératoire.

RÉSULTATS : Au total, 12183 opérations ont été effectuées en chirurgie plastique entre 2003 et 2013, dont 96 infections au site opératoire, pour un taux net d'infections opératoires s'élevant à 0,79\%. Les chirurgiens ont déclaré un taux d'infections de $0,49 \%$ après des implants. Pour ce qui est des opérations non liées à la chirurgie plastique, le taux d'infections au site opératoire variait entre $0,04 \%$ après des chirurgies des cataractes, et $13,36 \%$ après des hystérectomies abdominales à haut risque.

EXPOSÉ : Le taux d'infections après une chirurgie plastique au sein de l'établissement à l'étude était inférieur à $1 \%$. Ce taux était égal ou quelque peu inférieur au taux d'infections au site opératoire. Cependant, ces résultats ne tiennent pas compte des profils des taux d'infections liées aux interventions, à la saison, aux groupes d'âge ou au sexe. Pour approfondir le sujet, il faudrait mener une étude plus vaste.
T aunched in 2002, the Tracking Operations and Outcomes for LPlastic Surgeons (TOPS) is an initiative from the American Society of Plastic Surgery; it is designed to serve as a secure and confidential national database of plastic surgery procedures and outcomes in the United States. For a minimal fee, plastic surgeons can register, upload and compare their outcomes data (eg, complication in postmastectomy procedures, cardiac events in maxillectomy patients, range of motion, post-needle aponeurotomy, etc). To date, two publications have resulted from this database: one examining outcomes after use of acellular dermal matrix in implant/expander breast reconstruction (1); the second regarding complication rates in abdominoplasty and breast augmentation patients (2). At this point, however, there remain no comprehensive reports describing infection rates for plastic surgery procedures in the literature. Previous studies have reported hospital-acquired infection rates encompassing any and all surgical services, but have failed to provide infection rates specifically focused on plastic surgery (3-5).
Surgical site infections (SSIs) may lead to significant morbidity for affected patients, a need for secondary operations, prolonged hospital stays and increased utilization of health care resources. Self-reported data have been noted to be of considerable value in other surgical specialities for determining infection rates when other sources of information are lacking (6). Clinically, this is still considered to be superior to electronic means of recording infections, which are performed either through billing records or electronic surveillance systems. Studies have shown that electronic methods result in under-reporting due to the dependence on physician record keeping and on the medical coders' abilities to extract information from patient charts (7). Presently, the Ministry of Health requests infection rates from hospitals in Ontario for its public safety reporting website (Health Quality Ontario); however, this requirement does not include plastic surgery (8). Knowledge of the rates of SSI in plastic surgery can enable surgeons to better inform future patients, hospital administrators and third-party payers of these rates. Hence, the objective of the present

\footnotetext{
${ }^{1}$ Division of Plastic Surgery, Department of Surgery; ${ }^{2}$ Surgical Outcomes Research Centre, Department of Surgery; ${ }^{3}$ School of Rehabilitation

Sciences, Faculty of Health Sciences; ${ }^{4}$ Department of Clinical Epidemiology and Biostatistics, Faculty of Health Sciences, McMaster University, Hamilton, Ontario

Correspondence: Dr Achilleas Thoma, Division of Plastic Surgery, McMaster University, 206 James Street, Suite 101, Hamilton, Ontario L8P 3 A9. Telephone 905-523-0019, fax 905-523-0229, e-mail athoma@mcmaster.ca
} 
TABLE 1

Centers for Disease Control \& Prevention (Georgia, USA) criteria for surgical site infections (SSIs)

Superficial incisional SSIs must occur within 30 days after the operative procedure and involve only skin or subcutaneous tissue of the incision, and at least one of the following is present:

- Purulent drainage from the superficial incision.

- Organisms isolated from an aseptically obtained culture of fluid or tissue from the superficial incision.

- At least one of the following signs or symptoms of infection: pain or tenderness, localized swelling, redness, or heat - and the superficial incision is deliberately opened by the surgeon, unless culture of incision is negative.

- Diagnosis of superficial incisional SSI by the surgeon or attending physician.

The following are not reported as superficial incisional SSI: stitch abscess (minimal inflammation and discharge confined to the points of suture penetration), infection or an episiotomy or a neonate's circumcision site, infected burn wound, incisional SSI extending deep into the fascial and muscle layers.

Deep incisional SSIs must meet the following criteria: infection occurs within 30 days after the operative procedure if no implant (defined as a nonhuman-derived implantable foreign body) is left in place, or within 1 year if implant is in place and the infection appears to be related to the operative procedure and the infection involves deep soft tissues of the incision. In addition, at least one of the following is present:

- Purulent drainage from the deep incision.

- A deep incision spontaneously dehisces or is deliberately opened by a surgeon when the patient has at least one of the following signs or symptoms: fever, localized pain or tenderness, unless culture of the incision is negative.

- An abscess or other evidence of infection involving the deep incision is found on direct examination.

- Diagnosis of a deep incisional SSI by a surgeon or attending physician.

\section{TABLE 2}

Infection rates over an eight-year period at St Joseph's Healthcare Hamilton, Hamilton, Ontario

\begin{tabular}{lc}
\hline Procedure type & Infection rate, \% \\
\hline Plastic surgery & 0.79 \\
Implant-based plastic surgery & 0.49 \\
Low-risk laparoscopic cholecystectomy & 0.43 \\
Moderate-risk laparoscopic cholecystectomy & 0.90 \\
High-risk laparoscopic cholecystectomy & 0.74 \\
Caesarean section & 1.78 \\
Low-risk abdominal hysterectomy & 1.82 \\
Moderate-risk abdominal hysterectomy & 4.09 \\
High-risk abdominal hysterectomy & 13.36 \\
Cataract surgery & 0.04 \\
Low-risk hip arthroplasty & 0.63 \\
Moderate-risk hip arthroplasty & 1.24 \\
High-risk hip arthroplasty & 3.11 \\
Low-risk knee arthroplasty & 0.27 \\
Moderate-risk knee arthroplasty & 0.56 \\
High-risk knee arthroplasty & 2.39 \\
Open reduction and internal fixation of long bone fractures & 1.17 \\
Low-risk thoracotomy & 1.23 \\
Moderate-risk thoracotomy & 1.02 \\
High-risk thoracotomy & 1.57 \\
\hline
\end{tabular}

study was to conduct a review of all self-reported infections postplastic surgery at one centre. We believe that these data may encourage other hospital centres to evaluate their SSI rates in plastic surgery, which, in turn, will yield more comprehensive reports in the literature to accompany the confidential TOPS database.

\section{METHODS}

The present study was conducted at St Joseph's Healthcare Hamilton (SJHH, Hamilton, Ontario), an academic teaching hospital. SJHH provides tertiary, secondary and ambulatory health care services through referrals from the Hamilton-Niagara-Haldimand-Brant Local Health Integration Network, as well as the neighbouring regions of Halton, Kitchener-Waterloo and Norfolk. Ethics approval for the study was obtained from the Hamilton Integrated Research Ethics Board.

Criteria for nosocomial SSIs were defined according to the Centers for Disease Control \& Prevention (CDC, Georgia, USA) (Table 1) (9). SSI data were obtained from the Infection Prevention and Control office at SJHH. Infection statistics have been collected prospectively by the Infection Prevention and Control office since 2003. These data are primarily self-reported by surgeons, using the aforementioned CDC criteria, on a monthly basis irrespective of specialty. Furthermore, the Infection Prevention and Control office tracks patients who return to the hospital through the emergency room as a result of operative site infection; SSI is confirmed by the most responsible physician.

A retrospective review of all plastic surgery procedures performed in the main operating room at SJHH between January 1, 2003 and January 1, 2013 was conducted. Subsequently, the SSIs confirmed by the primary surgeon were recorded and the total operative SSI rates were calculated from these data. A subset of implant-based plastic surgery procedures were then identified. Implant-based procedures were defined as any procedure in which $\geq 1$ foreign bodies (eg, plate, implant or tissue expander) are inserted. Percutaneous procedures, such as those involving Kirschner wires without further opening of skin, were excluded.

SSI rates for other specialties, including general surgery, ophthalmology, gynecology, orthopedic surgery and thoracic surgery, were obtained for comparison from the same Infection Prevention and Control database. These procedures included laparoscopic cholecystectomy, Caesarean section, cataract surgery, hip and knee arthroplasty, long bone fracture fixation and thoracotomy.

Prospective infection surveillance data have been gathered routinely over an eight-year period (January 1, 2005 to January 1, 2013) for non-plastic surgery procedures. This information is collected by the Infection Prevention and Control office, but with risk stratifications for certain procedures. For example, for laparoscopic cholecystectomy, risk is determined using a three-item assessment: length of surgery $>2 \mathrm{~h}$; wound class I and II; and American Society of Anesthesiologists score $>2$ (Table 2).

Analysis included determining infection rates by dividing the total number of SSIs by the total number of plastic surgery procedures.

\section{RESULTS}

Between January 1, 2003 and January 1, 2013, a total of 12,183 plastic surgery operations were performed in the main operating room at SJHH. A total of 96 operative SSIs were identified over this 10-year period, corresponding to a total operative SSI rate of $0.79 \%$.

Data regarding the types of plastic surgery procedures performed before 2007 were unavailable. Between January 1, 2007 and January 1, 2013 (six-year period), 821 of 7326 plastic surgery operations involved implant-based procedures. These included breast augmentation, insertion of tissue expanders, exchange of tissue expanders for implants, open reduction and internal fixation of hand or facial fractures, and finger arthroplasty. Of these procedures, a total of four were reported to have SSIs: open reduction and internal fixation of a thumb proximal phalanx fracture; open reduction internal fixation of a middle phalanx; a finger arthroplasty of a metacarpophalangeal joint; and a 
TABLE 3

Plastic surgery infection rates according to year at St Joseph's Healthcare Hamilton, Hamilton, Ontario

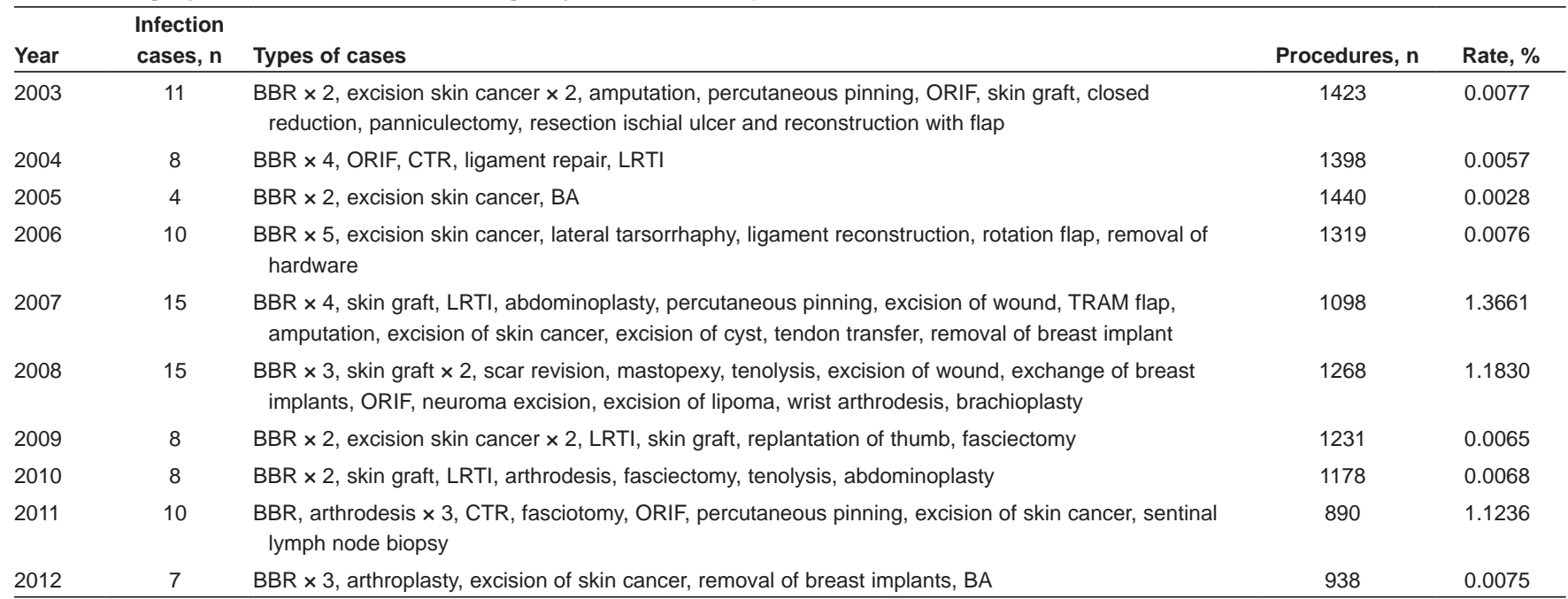

BA Breast augmentation; BBR Bilateral breast reduction; CTR Carpal tunnel release; LRTI Ligament reconstruction tendon interposition; ORIF Open reduction and internal fixation; TRAM Transverse rectus abdominis myocutaneous

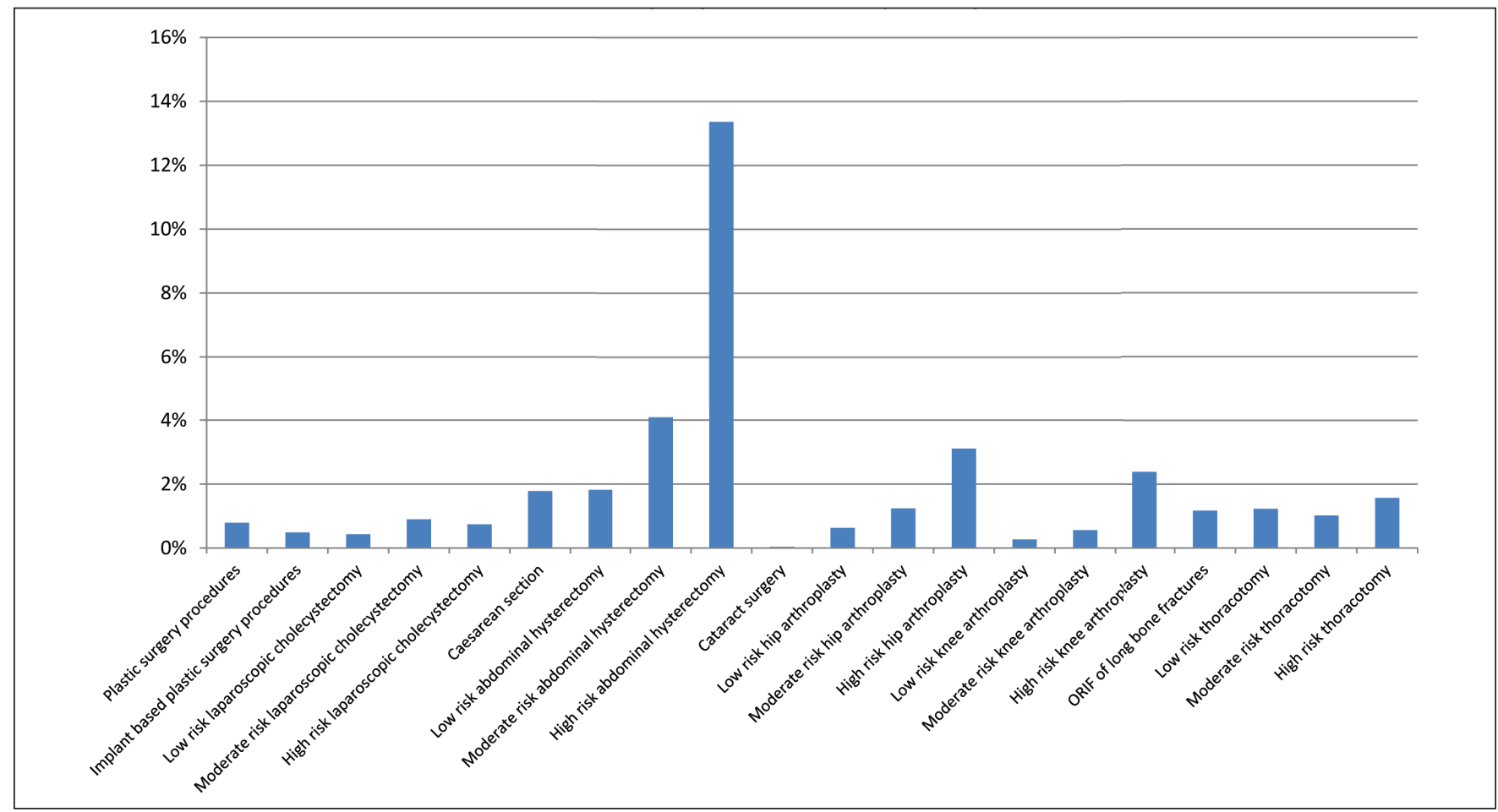

Figure 1) Infection rates over a 10-year period at St Joseph's Healthcare Hamilton, Hamilton, Ontario. ORIF Open reduction and internal fixation

bilateral breast augmentation. This resulted in a $0.49 \%$ self-reported SSI rate for implant-based procedures.

Over the 10-year period, annual plastic surgery operative SSI rates ranged from $0.0028 \%$ to $1.4 \%$ per annum. Bilateral breast reductions were the most frequently reported types of operations to have SSIs, although these procedures were considerably more frequent than other procedures performed at $\mathrm{SJHH}$, with approximately 130 bilateral breast reductions performed annually (Table 3 ).

For nonplastic surgery procedures between January 1, 2005 and January 1, 2013, operative SSI rates ranged from $0.04 \%$ for cataract surgery to $13.36 \%$ for high-risk abdominal hysterectomies (Table 2, Figure 1).

\section{DISCUSSION}

The rate of SSIs reported in the present study was far less than the overall rate of $16.7 \%$ at 30 days in a 1995 study by Andenaes et al (10) investigating elective plastic surgery procedures. Their criteria for wound infection was based on the postoperative wound condition defined on a scale that the authors had developed (a score of $\geq 4$ of 7 indicated an SSI). The difference in reported rate may also be due to the difference in definition of a SSI used for their study. It has been reported that $5 \%$ of all patients undergoing plastic surgery develop SSIs, accounting for $20 \%$ of health care-related infections. Surgical technique, suture type and placement of drains can influence infection rates (11). Some studies suggest that wound dressings may influence postoperative 
infection, particularly when pins or implants are used (12), whereas other studies suggest that dressings have no impact on SSIs (13).

Care must be taken when interpreting the data in the present study because data collected at this centre differing from other centres can likely be explained by variation in patient population, an observation that has been suggested in other studies (14). Variations could also be due to a lack of standardized definition of SSIs - while SJHH uses the CDC definition, others have used ad hoc scales.

The plastic surgery SSI rate at the study institution was generally equal to or less than non-plastic SSI rates. No patterns were identified in terms of types of procedures, nor were any patterns identified in monthly variances in infection rates over the 10 -year period.

These data are useful in providing information to patients, surgeons, hospitals and third-party payers regarding overall recent SSI rates in plastic surgery. Our report provides proof of concept of the feasibility of collecting such information through an administrative office.

One limitation germane to the present study was the results being based on surgeon-reported data. However, a representative from the Infection Prevention and Control office screens all patient charts and admission notes on surgical patients to identify additional patients who may have SSIs. The hospital representative searches for any surgical patient who registers or reregisters to the hospital to determine whether any subsequent visit to hospital is infection related. This effort enables the identification of missed patients, which mitigates the aforementioned limitation. Moreover, we did not collect data regarding the length of surgeries, patients' smoking status, perioperative antibiotics, surgical techniques, patient ages, comorbities, level of immunocompetency, treatment, recurrence, types of wound dressings or drain use. In addition, the surgeonreported data available to us did not include the severity of SSI, ward location, or the need for subsequent surgery or further hospital stays, aside from cases that were identified through the Infection Prevention and Control office. Another possible limitation of the present study was that some patients may have presented with SSIs to emergency departments outside SJHH or to primary health care providers, and were successfully treated conservatively elsewhere. Thus, these patients may not have been reported to the treating plastic surgeon and, consequently, were not included in the study. This discrepancy has the potential to introduce bias into the study results. For example,

\section{REFERENCES}

1. Alderman AK, Collins ED, Streu R, et al. Benchmarking outcomes in plastic surgery: National complication rates for abdominoplasty and breast augmentation. Plast Reconstr Surg 2009;124:2127-33.

2. Pannucci CJ, Antony AK, Wilkins EG. The impact of acellular dermal matrix on tissue expander/implant loss in breast reconstruction: An analysis of the Tracking Outcomes and Operations in Plastic Surgery database. Plast Reconstr Surg 2013;132:1-10.

3. Asaid R, Williams I, Hyde D, et al. Infection rates following hip and knee joint arthroplasty: Large referral centre versus a small elective-only hospital. Eur J Orthop Surg Traumatol 2013;23:165-8.

4. Lonjon G, Dauzac C, Fourniols E, et al. Early surgical site infections in adult spinal trauma: A prospective, multicentre study of infection rates and risk factors. Orthopaed Traumatol Surg Res 2012;98:788-94.

5. Wenzel RP, Osterman CA, Hunting KJ. Hospital-acquired infections: Infection rates by site, service and common procedures in a university hospital. Am J Epidemiol 1976;104:645-51.

6. Curtin P, Harty J, Sheehan E, et al. Self-reported complication rates following primary total hip arthroplasty in Ireland: Fact or fiction. Ir J Med Sci 2011;180:167-71.

7. Hollenbeak CS, Boltz MM, Nikkel LE, et al. 2011. Electronic measures of surgical site infection: Implications for estimating risks and costs. Infect Control Hospital Epidemiol 2011;32:784-90.

8. Health Quality Ontario, 2014. Patient Safety. <www.hqontario.ca/ public-reporting/patient-safety $>$ (Accessed June 15, 2014).

9. Centers for Disease Control \& Prevention. <www.cdc.gov/> (Accessed June 15, 2014). elderly patients who live in rural areas and have difficulty accessing transportation were more likely to be missed.

There were too few reported SSIs in the present study to draw conclusions regarding seasonal or time-of-year variations in SSIs, which may impact rates (15). It was not noted whether our patients were on the same postoperative unit as other surgical subspecialty patients, which may influence postoperative SSIs through exposure (16).

An additional limitation of the present study was that the data did not include patients who underwent their procedures in a minor procedure room setting. As such, patients who underwent procedures in the minor procedure room under local anesthetic alone through sameday surgery were not included. Because this population of patients comprises a large proportion of plastic surgeons' practices, this limitation is significant. Our hospital performs an average of 1872 plastic surgery procedures in the minor procedures room per annum, compared with 1218 plastic surgery procedures in the main operating room per annum; therefore, more than one-half of all plastic surgery procedures per year were missed in our analysis.

Recording and reviewing SSI rates can allow for measures to be taken to modify risk factors, leading to notable decreases in SSI rates over time (17). The process of prospective surveillance may have played a role in the low SSI infection rate. As more hospitals employ infection control offices to monitor and mitigate infection outbreaks, data from these offices may become more useful in understanding plastic surgery SSI rates on a widescale basis. This topic has become increasingly relevant in the United States because recently instituted quality-based penalty programs reduce Medicare payments to hospitals that report higher numbers of health care-associated infections (18). We present the rate of SSIs in our institution to prompt other investigators from across the country to consider this important quality of health care indicator as a clinical research activity.

DISCLOSURES: The authors have no financial disclosures or conflicts of interest to declare.

ACKNOWLEDGEMENT: The authors thank the staff at the Infection Prevention and Control office and SJHH Operating Room at St Joseph's Healthcare Hamilton (Hamilton, Ontario) for their cooperation in collecting data for this study.

10. Andenaes K, Amland P, Lingaas E, et al. A prospective, randomized surveillance study of postoperative wound infections after plastic surgery: A study of incidence and surveillance methods. Plast Reconstr Surg 1995;96:948-56.

11. McHugh SM, Hill ADK, Humphreys H. Intraoperative technique as a factor in the prevention of surgical site infection. J Hospital Infect 2011;78:1-4.

12. Lee CK, Chua YP, Saw A. Antimicrobial gauze as a dressing reduces pin site infection. Clin Orthop Relat Res 2012;470:610-5.

13. Walter CJ, Dumville JC, Sharp CA, et al. Systematic review and meta-analysis of wound dressings in the prevention of surgical-site infections in surgical wounds healing by primary intention. Br J Surg 2012;99:1185-94.

14. Van Dishoeck AM, Koek MBG, Steyerberg EW, et al. Use of surgical-site infection rates to rank hospital performance across several types of surgery. Br J Surg 2013;100:628-37.

15. Gruskay J, Smith J, Kepler CK, et al. The seasonality of postoperative infection in spine surgery. J Neurosurg Spine 2013;18:57-62.

16. Anwar M, Ferguson L, Awad Z, et al. The impact of mixing surgical subspecialty patients on wound infection rates. Eur Arch Otorhinolaryngol 2012;269:261-4.

17. Renaud A, Lavigne M, Vendittoli P. Periprosthetic joint infections at a teaching hospital in 1990-2007. J Can Chir 2012;55:394-400.

18. Peasah SK, McKay NL, Harman JS, et al. Medicare non-payment of hospital-acquired infections: Infection rates three years post implementation. Medicare Medicaid Res Rev 2013;3: E1-E13. 